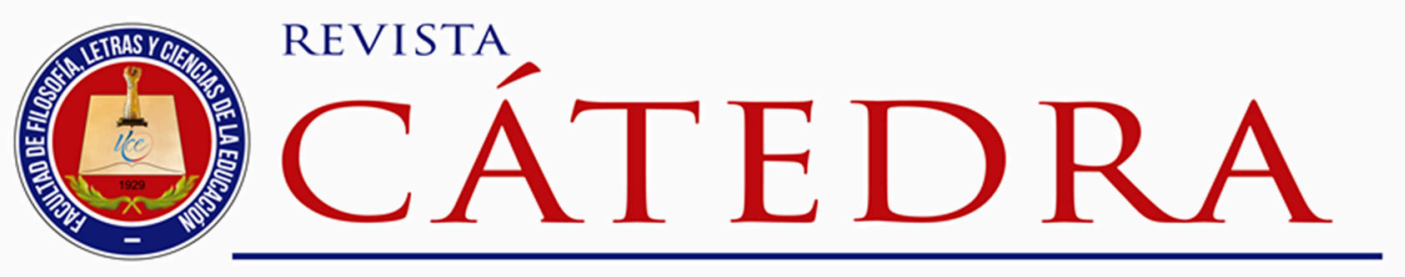

\title{
Desafíos de la educación virtual en Latinoamérica
}

\section{Challenges of virtual education in Latin América}

\author{
Pedro Cantú-Martínez \\ Universidad Autónoma de Nuevo León, México \\ cantup@hotmail.com \\ https://orcid.org/0000-0001-8924-5343
}

(Recibido: 07/12/2021; Aceptado: 10/12/2021; Versión final recibida: 23/12/2021)

Cita del artículo: Cantú-Martínez, P. (2022). Desafíos de la educación virtual en Latinoamérica. Revista Cátedra, 5(1), 71-79.

\section{Resumen}

Este artículo tiene como intención manifestar y exhibir los retos de la educación virtual en Latinoamérica producto de los adelantos en las tecnologías de comunicación e información que hoy en día se despliegan internacionalmente, y que además se vieron acelerados tras la pandemia del covid-19. Se conocía, que la educación virtual conllevaría un cambio en los procesos de enseñanza y aprendizaje, pero no de esta manera que predominó, donde se reveló en toda su contextualización, ya que se caracterizó por el abandono en el acompañamiento y tutelaje de los estudiantes. En América Latina la educación se transfiguró de las instituciones a los hogares de los estudiantes, conllevando experiencias educativas mayormente negativas más que positivas para una gran cantidad de estudiantes de todos los niveles escolares. Esto se debió a la gran desigualdad social prevaleciente en Latinoamérica, creando una nueva categorización social, como son los inforricos y los infopobres. En otras palabras, entre los que pueden acceder y no a la educación virtual. Lo anterior, mostró nuevamente las grandes diferencias sociales, económicas y de educación que aún existen en América Latina. Por último, esto no favorece desafortunadamente a la alfabetización de las personas, como tampoco al derecho universal de la educación.

\section{Palabras clave}

Desigualdad social, educación, educación virtual, pobreza.

\section{Abstract}

The purpose of this article is to show and exhibit the challenges of virtual education in Latin America as a result of the advances in communication and information technologies that are currently being deployed internationally, and which were also accelerated after the COVID- 
19 pandemic. It was known that virtual education would lead to a change in the teaching and learning processes, but not in the way it prevailed, where it was revealed in all its contextualization, since it was characterized by the abandonment in the accompaniment and tutoring of students. In Latin America, education was transfigured from the institutions to the students' homes, leading to mostly negative rather than positive educational experiences for a large number of students at all school levels. This was due to the great social inequality prevailing in Latin America, creating a new social categorization, such as the inforricos and the infopobres. In other words, between those who can and cannot access virtual education. The above, showed again the great social, economic and educational differences that still exist in Latin America. Finally, this unfortunately does not favor people's literacy, nor does it favor the universal right to education.

\section{Keywords}

Social inequality, education, virtual education, poverty.

\section{Introducción}

Este artículo tiene como propósito demostrar y exponer los desafíos de la educación virtual en Latinoamérica producto de los avances en las tecnologías de comunicación e información que en la actualidad se ostentan a nivel internacional, y que además son generados por los efectos de la globalización, y que hoy en día tras la pandemia del covid-19 ha llegado como una revolución a la educación - irrumpiendo - y generando cambios sustanciales en los procesos de enseñanza aprendizaje, tanto a maestros, estudiantes y personal administrativo (Cantú-Martínez, 2021). Ante esto, ha sido sumamente relevante el papel que han jugado las instancias gubernamentales en materia de educación, como también la iniciativa privada, al hacer partícipes a la sociedad en general de este abrupto cambio conforme la pandemia de covid-19 transcurría y la misma tecnología progresaba vertiginosamente ante la demanda de todas las instituciones del sector de educación de incorporar estas nuevas tecnologías para seguir brindando acceso a la educación de grandes masas de alumnos de distintos niveles de preparación.

En distintos países de América Latina la educación trasmutó de los recintos de las instituciones a los hogares de los estudiantes, como también los maestros y trabajadores administrativos trasladaron las labores y responsabilidades a la intimidad de las casas; evolucionando de una educación de impartición presencial a una no presencial, y en otros casos a la semipresencial. El uso del internet se convirtió en una plataforma idónea para trasmitir conocimiento entre las personas, y aunque esto todavía es debatible particularmente en el proceso de enseñanza aprendizaje y así garantizar las competencias y habilidades de los estudiantes, fue la respuesta más idónea encontrada con el fin de establecer los vínculos en la educación y continuar con la preparación educativa de los estudiantes.

Por otra parte, este escenario puso de manifiesto las deficiencias estructurales como desigualdades existían en los sistemas educativos y entorno socioeconómico de las personas. De esta manera, se dejó a la deriva a un nutrido grupo de estudiantes en toda la región latinoamericana por no contar con las capacidades y recursos económicos - que posibilitarán - incorporar los equipos e infraestructura necesaria para mantener la continuidad de sus aprendizajes, quedando invisibles antes las autoridades de educación y sociedad misma.

Con lo cual, se aleja la educación de la función pública, ya que la educación virtual y quienes la impulsan están muy distantes de los preceptos de funcionabilidad sincrónica y preceptora del proceso de aprendizaje que garantice las competencias que los estudiantes deben ostentar para avanzar en la formación personal y así integrarse al espacio social 
laboral. El presente escrito pretende además ofrecer una reflexión -desde la postura de la bioética hermenéutica- sobre este suceso y exhibir los retos que la educación virtual plantea conjuntamente con sus medios en la forma de erigir un ser humano, en un entorno que está afectando el modo de ser, existir y pensar de toda la sociedad en el mundo. Y en el que, además, subsiste una brecha digital que ahora definirá la distancia entre las personas que pueden acceder a una educación de aquellas que no lo pueden hacer por motivos y/o causas económicas y culturales, creando así, nuevas estructuras de poder definidas en derredor del acceso a la educación virtual (Venegas y Green, 2018).

\section{Desarrollo}

Con la finalidad de comprender las implicaciones sociales y desafíos que se plantean para las sociedades latinoamericanas en materia de educación virtual, se hace necesario clarificar el panorama de su uso y la experiencia que se ha tenido como medio emergente durante esta pandemia de covid-19, que lejos de ser un valor agregado que permitiera acceder a los contenidos educativos se transformó en experiencias educativas negadas para un gran volumen de estudiantes. Por lo tanto, se adentrará en esta temática y emprenderá una línea discursiva sobre el papel de la educación, la realidad en Latinoamérica y el contexto de la educación virtual en la región de Latinoamérica.

\subsection{Papel de la educación en la sociedad}

$\mathrm{Al}$ retomar el discurso sobre la educación se hace pertinente considerar con gran atención los fundamentos de esta. Esencialmente desde el fecundo diálogo que debe subsistir entre el mentor y el educando, que de acuerdo con Paulo Freire permite meditar lo deliberado, y se circunscribe a la actividad de instruir en el marco de la contextualización social en que se existe (Guichot, 2006). Esto es, como Mires (1996) comentó, es en la formación de educar a las personas que se entretejen los hilos de la dialéctica y la complejidad de la realidad, donde emanan consideraciones que pueden ser desiguales o de concordancia entre los seres humanos. Es así, que la educación -como instrumento social- está consignada al desarrollo de las personas, fundamentalmente en tres ámbitos como son la capacidad intelectual, moral y a la socialización del ser humano en función del contexto sociocultural en que se vive. Con ello, la educación se erige como uno de los factores que más inciden en el progreso de las sociedades, esto es la educación es ineludible en todos los sentidos (OCDE/CEPAL/CAF, 2016). De tal manera, que la educación se instituye en un medio para el desarrollo de todas las personas y adicionalmente conlleva la transformación de la sociedad, en la cual se busca establecer una solidaridad de orden social que caracterice a sus miembros.

Por este motivo, en todas las naciones del mundo se reconoce el derecho que ostenta toda persona a la educación; lo cual es señalado en el artículo 26 de la "Declaración Universal de Derechos Humanos" (Naciones Unidas, 1948) que marca en los puntos 1 y 2 lo siguiente:

1. Toda persona tiene derecho a la educación. La educación debe ser gratuita, al menos en lo concerniente a la instrucción elemental y fundamental. La instrucción elemental será obligatoria. La instrucción técnica y profesional habrá de ser generalizada; el acceso a los estudios superiores será igual para todos, en función de los méritos respectivos.

2. La educación tendrá por objeto el pleno desarrollo de la personalidad humana y el fortalecimiento del respeto a los derechos humanos y a las libertades fundamentales; favorecerá la comprensión, la tolerancia y la amistad... 
Desde esta configuración, la educación retoma un papel de formación, ilustración, interpretación y revelación para las personas, lo cual les permite ser libres, objetivas e imparciales en su actuar. Esto es, desde una perspectiva positivista la educación tiene como fin último erigir un ser humano con las cualidades y competencias necesarias para afrontar su vida futura y sortear los obstáculos con las capacidades intelectuales y morales que le exija el medio social (Durkheim, 1989). Particularidad -que hoy en día- se ratifica también en la meta número 4.3 del objetivo de desarrollo sostenible 4 que atiende los aspectos de la educación, y que indica que se debe aumentar la cantidad de personas en el mundo con las competencias suficientes para integrarse a la sociedad, y así acceder a un trabajo decente (Naciones Unidas, 2021). Que también ha inscrito que el progreso socioeconómico de una sociedad se finca en la educación de las personas, y permite así abreviar la pobreza social en que subsiste un gran número de personas. En este sentido, Munari (1994) expresaba desde la década de los noventas del siglo pasado que la educación debería considerársele como una institución del bien común. Cuya argumentación se sostiene en la obligatoriedad de la educación para todas las personas, como también que esta debe otorgarse en el marco de la gratuidad, preceptos que se articulan en la igualdad y en lo establecido por la “Declaración Universal de Derechos Humanos” promulgada en 1948.

\subsection{Situación en Latinoamérica}

Las condiciones de los entornos de vida en América Latina manifiestan brechas estructurales caracterizadas por la presencia de distintas crisis sociales, en las que tanto la igualdad como la sostenibilidad de las economías se han visto afectadas postergando la posibilidad de acceder por las personas a una condición de bienestar general. Esto conlleva que todas las naciones latinoamericanas confronten distintos y muy variados desafíos sociales, económicos y ambientales, que evidencian desigualdad, marginación, pobreza y vulnerabilidad social. En este sentido la Comisión Económica para América Latina y el Caribe -por sus siglas CEPAL- (2021) expresa lo siguiente refiriéndose a Latinoamérica:

\footnotetext{
El COVID-19 llega a una región marcada por una matriz de desigualdad social, cuyos ejes estructurantes - el estrato socioeconómico, el género, la etapa del ciclo de vida, la condición étnico-racial, el territorio, la situación de discapacidad y el estatus migratorio, entre otros- generan escenarios de exclusión y discriminación múltiple y simultánea que redundan en una mayor vulnerabilidad ante los efectos sanitarios, sociales y económicos de esta enfermedad (p. 13).
}

Todo lo anterior adyacente a un malestar prevaleciente -de orden general- en los países latinoamericanos con relación a las políticas públicas, que por lo habitual se acompañaban de actos de protesta y resistencia civil, para demandar llanamente justicia de carácter social. Situación que Cetrángolo y Curcio (2017) lo atribuían a "la desigualdad de oportunidades al comienzo de la vida y durante el ciclo de la educación, que a su vez impacta de manera significativa en las posibilidades de lograr niveles más elevados de productividad, desarrollo y empleo de calidad" (p. 7). Esto se proyecta, según la CEPAL (2021), de manera muy nítida durante el lapso de 1990 al 2020 en Latinoamérica donde se incrementó la pobreza extrema del $7.8 \%$ al $11.3 \%$-que representa 78 millones de personas en pobreza extrema- en tanto la población en pobreza aumentó en este mismo período de $27.8 \%$ a $30.5 \%$ que representa 209 millones de personas en esta condición. Esto es equivalente -en el caso de la pobreza extrema- a considerar la población colombiana y venezolana de manera conjunta; mientras que la representada por la pobreza sería semejante a congregar a la totalidad de la población de los siguientes países: Argentina, Chile, Paraguay, Bolivia, Perú, Ecuador, Colombia y Venezuela. De esta manera Blanco (2006) expresa que la región latinoamericana se "caracteriza por tener sociedades muy desintegradas y fragmentadas debido a la persistencia de la pobreza y a la gran desigualdad en la distribución de los

Licencia Creative Commons Atribución 4.0 Internacional (CC BY 4.0)

Revista Cátedra, 5(1), pp. 71-79, enero-junio 2022. e-ISSN:2631-2875

https://doi.org/10.29166/catedra.v5i1.3487 
ingresos, lo cual genera altos índices de exclusión" (p. 1). Esto ha implicado también que la situación en la actualidad de la educación en la región latinoamericana este a la zaga de las exigencias globales para constituir personas con competencias y habilidades para integrarse a la modernidad prevaleciente en la sociedad. En este contexto lo antes advertido denota un esquema sumamente complejo para los países latinoamericanos ya que los requerimientos en materia de preparación educativa no se cumplen, en tanto las políticas en materia de educación se tornan también disonantes con el contexto social prevaleciente en el mundo como en sus circunstancias nacionales de manera particular (Martínez, 2009).

Por consiguiente, De la Cruz (2017) asevera que la cimiente de toda política - regional o nacional - debe ser esencialmente equitativa e inclusiva, y en este caso la de carácter educativa, debe brindar la posibilidad a toda persona de acceder a una vida plena y con dignidad que le permita edificar su trayectoria de vida, como también tomar sus propias decisiones en la vida. Situación que es sumamente cuestionable con el escenario regional que poseemos. Es así que las condiciones prevalecientes dificultan una educación inclusiva para todas las personas, y donde todavía es ostensible encontrar grandes brechas en la calidad de la educación impartida.

\subsection{Escenario de la educación virtual en Latinoamérica}

La educación de manera general, se ha visto afectada por los propios cambios de la modernidad y del avance de las tecnologías de información, como también de comunicación que han influido en los procesos de enseñanza aprendizaje. Sin embargo, este interés promovido en el discurso previo a la pandemia de COVID-19, plantea ahora muchas preguntas tras la aparición emergente de la educación virtual. Ante esto, surgen distintas interrogantes $i$ Qué retos existen en América Latina para introducir una educación virtual inclusiva y comprometida? ¿Qué desafíos del ámbito teórico y metodológicos plantea este nuevo escenario virtual? ¿Se considerarán las experiencias virtuales que ha dejado la pandemia de COVID-19, o llanamente se ignorarán? ¿Se puede aspirar a esta modalidad virtual cuando en el contexto de la región latinoamericana persisten aún grandes rezagos estructurales de carácter social, y la calidad educativa es además desemejante entre los miembros de una misma sociedad?

Estas preguntas y otras más, brotan en la actualidad en el campo de la educación, "en un momento en el que las decisiones políticas requieren con más urgencia que nunca, conocimientos pertinentes y sustantivos sobre lo escolar, lo educativo, y la diversidad de actores implicados" (Gluz, Lima y Elías, 2020, p. 6). Sin lugar a dudas, esto emerge en el contexto social latinoamericano porque las instituciones educativas -contrariamente a sus propósitos substantivos- dejaron de ser proveedoras del espacio para ejercer prácticas educativas igualitarias -cavilando muy propositivamente- y donde se vulneró el derecho a la educación. Al reflexionar lo anterior, Gluz (2020) comenta que la crisis sanitaria develó una pobreza educativa de orden institucional, donde además fue ostensible una educación con "servicios específicos y devaluados para los grupos poblacionales vulnerados, diferentes de aquellos a los que acceden los grupos privilegiados" (p. 15). Por lo cual, las deficiencias estructurales se agudizaron, y que tienen su génesis en las crisis económicas y sociales previas, que además han persistido en el tiempo.

En este marco de referencia, para todos los países latinoamericanos conllevó problemáticas de orden institucional con una gran cantidad de contradicciones, donde la transición urgente hacia lo virtual genero eventualidades, principalmente de carácter financiero al carecer de la infraestructura adecuada. Por otra parte, creó una crisis tanto para los maestros y estudiantes, los cuales no ostentaban las habilidades ni la capacidad para trasladar las actividades -laborales y académicas- a los hogares; como tampoco se evaluó que hubiera más de un miembro de la familia que estudiase y/o con capacidades diferentes (Cantú-Martínez, 2021). En otras palabras: "Se reemplazó el aula de clases por espacios 
emergentes: dormitorio, comedor, sala, cuarto de estudio u otros similares; el contacto social entre compañeros de clases, amigos o docentes se limitó únicamente al contacto familiar" (Aguilar, 2020, p. 217). Lo anterior, dejó a relieve las brechas digitales como también las socioeconómicas que mostraban las familias de maestros como estudiantes. Y en particular, en la formación académica de los estudiantes pesarán consecuencias negativas donde será ostensible un descenso aún mayor de la calidad educativa -si es que se contaba con ella- por la implementación de la educación virtual, ya que -a nuestro parecer y experiencia- no garantiza está el apropiamiento de competencias, habilidades y destrezas, siendo únicamente de orden demostrativo. Lo que contradice el supuesto de Freire (1997) al aseverar que es la práctica donde "se confirman, se modifican o se amplían en los saberes" (p. 24).

Pero, además fue patente como los profesionales de la educación no eran considerados en esta planeación de educación virtual, claudicando ante los profesionales de la informática que se erigieron como quienes demarcarían -y lo hicieron-el rumbo de la educación de todo un país, inclusive conteniendo e ignorando las voces de los educadores de forma completa. Al convertir a los maestros de todos los niveles en simples repetidores de conocimientos, y en otros casos como simples actores y actrices de una escenificación llamada educación, donde se soslaya todo proceso para comprobar si el estudiante fue capaz de obtener una destreza o información, que garantice la transformación de este y le permita avanzar en su desarrollo como sujeto social (Aguilar, 2020). Se sabía, que la educación virtual vendría a cambiar los procesos de enseñanza y aprendizaje, pero no de esta manera que irrumpió, donde se manifestó en toda su expresión, ya que se caracterizó por el abandono en el acompañamiento y tutelaje de los estudiantes, no atendiendo las necesidades y problemáticas particulares de los escolares en los procesos de los aprendizajes. Igualmente se "ha perdido con ella, por ejemplo, el contacto directo o real con las personas, socializar, la relación que se tiene cara a cara y los sentimientos y expresiones espontáneas, ausencias que no aportan a lo humano" (Sepúlveda-Romero, 2019, p. 100).

Pero, sobre todo, se ignoró por completo los cuatro elementos fundamentales para el aprendizaje: "elementos del medio ambiente (sonido, luz, temperatura y el mobiliario), elementos emocionales (motivación, persistencia, responsabilidad, estructura), elementos sociológicos y físicos (potencial de percepción, ingesta, hora, movilidad) éstos determinan la habilidad, procesamiento y retención de información, valores, hechos y conceptos" (Castro y Guzmán de Castro, 2005, p. 86). Como también, hubo una desautorización silenciosa, del reconocimiento de los estilos de aprendizaje que pueden estar presentes en los estudiantes, como son el perfil activo, reflexivo, teórico y pragmático, como lo hacen saber Alonso, Gallegos y Honey (1994) y Cantú-Martínez y Rojas-Márquez (2018). Definitivamente, por estos juicios que hemos hecho saber otros profesionales de la educación, como Nieto (2012) se han pronunciado críticamente y consideran esta educación virtual una "parafernalia informática y comunicativa como un bien sucedáneo o sustituto" (p. 143), de características obsoletas que pretende sustituir la educación tradicional.

\section{Consideraciones finales}

Actualmente en América Latina existe una educación diferenciada ahora por los mecanismos de la virtualización y digitalización, como resultado de la contingencia sanitaria de COVID-19, donde se ha constatado que una gran parte de la sociedad ha sido excluida del acceso a la educación, provenientes estos de sectores caracterizados por ser humildes y de escasos recursos económicos, contando con distintas expresiones y particularidades en cada nación latinoamericana. 
Estos atributos comunes compartidos por distintos núcleos poblacionales a lo largo y ancho de Latinoamérica, han dado visibilidad de nueva cuenta de las fallas estructurales existentes en materia de educación, en la que subsisten aún muchas personas. De esta manera, se trastoca el fundamento señalado por Simón Rodríguez de una educación para todos, cuya argumentación sostiene que la educación es el soporte para edificar una sociedad más justa e igualitaria (García, 2010). Además, la educación virtual en la región latinoamericana está dando origen a un nuevo fenómeno social - que sustentado en las particularidades de estas poblaciones que se ha hecho mención - se puede denominar analfabetismo informático, producto del vacío existente para acceder y saber usar esta tecnología, y así agenciarse una educación pública por las personas de escasos recursos. Lo que ha creado una nueva categorización social como son los inforricos e infopobres, donde los primeros son los que poseen acceso a internet y cuentan con el equipamiento adecuado, mientras los segundos permanecen segregados de estas tecnologías (Cantú-Martínez, 2021).

De la mano de lo anterior, aún persisten más interrogantes que se constituyen en los desafíos debatibles a superar por la educación virtual para su correcta implementación en América Latina, como son: ¿Es lo mismo la enseñanza que la demostración virtual de contenidos? ¿Aprender virtualmente es solo cultivar contenidos de exposición teórica? ¿La educación virtual garantiza las competencias de los estudiantes? ¿Los estudiantes en su inmadurez vislumbran que están en una educación virtual con ambientes colaborativos y críticos acerca del conocimiento? ¿La comunicación mediante la virtualidad educativa supera la comunicación entre las personas cara a acara? ¿La educación virtual favorece a la sociabilidad de las personas? ¿La educación virtual abona a los derechos humanos de las personas? ¿La educación virtual garantiza la sustentabilidad social? ¿La educación virtual es una educación autodidacta? ¿O bien, la educación virtual es otro artificio social de poder y control político?

Finalmente, hagamos una reflexión profunda, reservada y honesta. ¿La educación virtual favorece al beneficio de la alfabetización de las personas, como también al derecho universal de la educación que asiste a toda persona en Latinoamérica? 


\section{Bibliografía}

Aguilar, F.R. (2020). Del aprendizaje en escenarios presenciales al aprendizaje virtual en tiempos de pandemia. Estudios Pedagógicos, 46(3), 213-223.

Alonso, C., Gallegos, D. y Honey, P. (1994). Los Estilos de Aprendizaje Procedimiento de Diagnóstico y Mejora. España. Ediciones Mensajero S.A.

Blanco, R. (2006). La equidad y la inclusión social: uno de los desafíos de la educación y la escuela hoy. REICE-Revista Electrónica Iberoamericana sobre Calidad, Eficacia y Cambio en Educación, 4(3), 1-15.

Cantú-Martínez, P.C. (2021). Educación superior durante y tras la pandemia de COVID-19 examinada mediante la bioética hermenéutica. En: P. Quezada (Coord.) SARS-COV2. COVID-19. La pandemia: reflexión y retos desde la bioética y la tanatología. (pp. 7696). México. Fondo Editorial de Nuevo León el Instituto de Investigaciones Jurídicas y Docencia de la Administración Pública del Estado de Nuevo León.

Cantú-Martínez, P.C. y Rojas-Márquez, J.M. (2018). Estilos de aprendizaje: La experiencia de la Escuela Preparatoria Técnica Médica en la Universidad Autónoma de Nuevo León, México. Revista Electrónica Educare, 22 (2), 1-8.

Castro, S. y Guzmán de Castro, B. (2005). Los estilos de aprendizaje en la enseñanza y el aprendizaje: Una propuesta para su implementación, Revista de Investigación, 58, 83-102.

Cetrángolo, O. y Curcio, J. (2017). Financiamiento y gasto educativo en América Latina. Santiago. CEPAL y Ministerio de Asuntos Exteriores de Noruega.

Comisión Económica para América Latina y el Caribe (2021). Panorama Social de América Latina, 2020. Santiago. CEPAL.

De la Cruz, G. (2017). Igualdad y equidad en educación: retos para una América Latina en transición. Educación, 26(51), 159-178.

Durkheim, E. (1989). Educación y Sociología. México. Colofón.

Freire, P. (1997). Pedagogía de la autonomía. Saberes necesarios para la práctica educativa. México. Siglo XXI Editores.

García, B. (2010). Pensamiento de Simón Rodríguez: la educación como proyecto de inclusión social. Revista Colombiana de Educación, 59, 134-147.

Gluz, N.B. (2020). Educación y desigualdades: desafíos para las políticas públicas en tiempos de pandemia. En: N.B. Gluz, C.M. Lima y R. Elias (Coords). Estado y derecho a la educación en América Latina. Desafíos para la investigación educativa a partir de la pandemia. (pp. 9-20). Buenos Aires. CLACSO.

Gluz, N.B., Lima, C.M. y Elias, R. (2020). Estado y derecho a la educación en América Latina. Desafíos para la investigación educativa a partir de la pandemia. Buenos Aires. CLACSO.

Guichot, V. (2006). Historia de la educación: reflexiones sobre su objeto, ubicación epistemológica, devenir histórico y tendencias actuales. Revista Latinoamericana de Estudios Educativos, 2(1), 11-51.

Martínez, A. (2009). La educación en América Latina: un horizonte complejo. Revista Iberoamericana de Educación, 49, 163-179. 
Mires, F. (1996). La revolución que nadie soñó o la otra posmodernidad. Caracas. Editorial Nueva Sociedad.

Munari, A. (1994). De la aventura de la OIE a los principios educativos de Piaget. Perspectivas-Revista de Educación Comparada, 24(2), 315-332.

Naciones Unidas (1948). La Declaración Universal de Derechos Humanos. Recuperado de https://www.un.org/es/about-us/universal-declaration-of-human-rights

Naciones Unidas (2021). Objetivo 4: Garantizar una educación inclusiva, equitativa y de calidad y promover oportunidades de aprendizaje durante toda la vida para todos. Recuperado de https://www.un.org/sustainabledevelopment/es/education/

Nieto, R.A. (2012). Educación virtual o virtualidad de la educación. Revista Historia de la Educación Latinoamericana, 14(19), 137-150

OCDE/CEPAL/CAF (2016), Perspectivas económicas de América Latina 2017: Juventud, competencias y emprendimiento. Paris. OECD Publishing.

Sepúlveda-Romero, M.E. (2019). Humanización del acto de la retroalimentación en la educación virtual. Revista Virtu@lmente, 7(1), 95-115.

Venegas, L.K. y Green, L.V. (2018) Medios interactivos: la brecha digital como agenciadora de la construcción de subjetividades. Educación y Ciudad, 35, 115-124.

\section{Autor}

PEDRO CÉSAR CANTÚ-MARTÍNEZ, cuenta con Doctorado en Ciencias Biológicas, Maestría en Salud Pública y Licenciatura en Biología, por parte de la Universidad Autónoma de Nuevo León (UANL). Además, cuenta con un Doctorado Honoris Causa por parte de la Organización Internacional para la Inclusión y Calidad Educativa (OIICE). Profesor de Tiempo Completo Titular "B" adscrito a la Facultad de Ciencias Biológicas de la UANL. También colabora en el Instituto de Investigaciones Sociales de la UANL y en el Instituto de Investigaciones en Bioética, A.C., donde es miembro invitado del claustro de académicos.

Es miembro del Sistema Nacional de Investigadores Nivel I en el área de ciencias sociales y cuenta con Perfil PRODEP. Es miembro el Cuerpo Académico UANL-181 de Ciencias Exactas y Desarrollo Humano que se encuentra Consolidado. Su línea de investigación es calidad de vida, desarrollo humano sustentable y ambiental, bioética y salud pública en la que ha dirigido tesis de posgrado y licenciatura. Ha colaborado en la Organización Panamericana de la Salud, en la Comisión de Salud Fronteriza México-Estados Unidos, entre otras organizaciones. Pertenece a la Red Mexicana de Educación Bioética adscrita a la UNESCO, Red de Investigación Educativa de la UANL, Red de Desarrollo Sostenible y Medio Ambiente en América Latina, Academia Nacional Mexicana de Bioética, Academia Universitaria para el Desarrollo Sustentable de la UANL y Red Iberoamericana de Docentes. Cuenta con la publicación de 22 libros, más 51 capítulos e innumerables artículos en el área de su especialidad, producto de los proyectos de investigación y formación de recursos humanos. 